\title{
Urbanismo bioclimático en Chile: propuesta de biozonas para la planificación urbana y ambiental
}

\author{
Luz-Alicia Cárdenas-Jirón. Universidad de Chile, Santiago, Chile. \\ Luis Morales-Salinas. Universidad de Chile, Santiago, Chile.
}

RESUMEN | El urbanismo bioclimático recrea la visión de ecosistema urbano para encauzar una urbanización amigable con el medioambiente, recordando el funcionamiento de los ciclos de elementos abióticos: el agua, el aire, los suelos y la energía. El disfuncionamiento de ellos por la acción antrópica ha producido patologías urbanas que se abordan mediante los planes de acción y regulación estatal. Considerando que el clima es un factor clave en el funcionamiento de los ciclos, se presenta una clasificación climática territorial chilena con un propósito práctico: aportar información accesible a planificadores y proyectistas urbanos; una información útil para estimar demanda energética y emplear recursos climáticos en planificación urbana y ambiental. La metodología consistió en levantar bases de datos de estaciones meteorológicas, interpolar y georreferenciar mediante un Sistema de Información Geográfica. Los resultados entregan unidades territoriales homogéneas que agregan espacialmente valores meteorológicos, intervinientes en los subsistemas del urbanismo bioclimático, complementando la actual norma chilena (NCh1079.Of2008).

PALABRAS ClAVE | urbanismo, medioambiente, planificación urbana.

ABSTRACT | Bioclimatic urbanism recreates an urban ecosystem vision to channel a type of urbanization that is friendlier with the environment. It recalls how the natural abiotic element such as water, air, ground and energy, operate in a cyclical way. Dysfunction of those cycles by anthropogenic actions has provoked urban pathologies that have been tackled by state action plans and regulations. Given that climate is a key factor in those cycles, a territorial climatic zoning is proposed to give accessible information for urban planners and project practitioners. Information is useful for estimating energy demand and applying climatic resources in urban and environmental planning. The methodology consisted in leveraging databases of meteorological stations along Chile, which were interpolated and geo-referenced through Geographical Information Systems. Results deliver homogeneous territorial units that spatially aggregate meteorological values, which are influential in the bioclimatic urbanism cycles, complementing the current Chilean normative (NCh1079.of2008).

KEYWORDS | urbanism, environment, urban planning. 


\section{Introducción}

El urbanismo bioclimático, un término acuñado por Higueras (1998) para referirse a las relaciones del ecosistema urbano, tiene por objetivo general comprender el funcionamiento del medioambiente construido. En particular, estudia estrategias de acondicionamiento climático aplicables a modelos urbanos territoriales y compatibles con el medioambiente natural. En ese marco, analiza con un enfoque ecosistémico los ciclos ecológicos urbanos -el ciclo atmosférico, el ciclo hidrológico, el ciclo de la materia y, finalmente, el ciclo energético- y sus patologías, a fin de abordar la configuración espacial y construcción de la ciudad de un modo más sostenible (Bettini, 1998; Higueras, 2006). Higueras plantea que, para abordar las patologías de los ciclos ecológicos urbanos, se deben incluir en la planificación urbana y en los proyectos de urbanismo recursos naturales del medioambiente, como aguas lluvias, brisas, vientos, energía solar. Tal planteamiento remite a preocupaciones de larga data en la planificación de ciudades y territorios: el arquitecto urbanista Fariña Tojo (2001) recuerda un principio clásico, mencionado ya en 1909 por "sir Raymon Unwin, el maestro de urbanistas", quien afirmaba que el planificador urbano deberá aproximarse al terreno con reverencia, captando con sensibilidad las sugerencias que el mismo le ofrezca. Indicaciones como el clima, la topografía, el viento, la vegetación, etcétera, son de gran valor para el proyectista, quien tiene el deber de interpretarlas correctamente para evitar proponer artefactos ineficientes y desvinculados del lugar.

Casi un siglo después, Girardet (1996) refuerza esa postura mediante el concepto de "ciudad sustentable", realzando con ello la visión ecosistémica de la ciudad; vale decir, las relaciones que el medioambiente construido establece con su medioambiente natural, o bien la relación entre los seres vivos (componentes bióticos) y elementos inertes (componentes abióticos). En un ecosistema urbano circulan la materia, la energía y la información, pero, a diferencia de un ecosistema natural, la ciudad no cumple un requisito fundamental: cerrar el ciclo metabólico. Una de las peculiaridades en el funcionamiento de los flujos en la ciudad es el extenso recorrido horizontal de los recursos y desechos que explotan otros ecosistemas lejanos, produciendo desequilibrios ecológicos territoriales en escala planetaria.

Girardet (2004) propone una visión sanadora, postulando el metabolismo circular como contrapropuesta al metabolismo lineal, que refleja el modo de producción predominante en las ciudades. En efecto, el metabolismo lineal se caracteriza por el excesivo consumo de recursos y descargas de residuos de forma ilimitada al medioambiente, natural o construido, proceso en que se ignora progresivamente el deterioro gradual del planeta tierra. El metabolismo circular consiste en el cierre del ciclo de la naturaleza; por ejemplo, en reutilizar los residuos, disminuir las cargas contaminantes en suelos, aire y agua, reducir el consumo de recursos, lo que supone un cambio en los patrones de producción y consumo en las ciudades. Esto implica cambiar las relaciones contemporáneas entre la ciudad y su medioambiente, transitar desde ser un ente parasitario a una relación simbiótica, o de apoyo mutuo. El autor propone transformar la concepción tradicional de "ciudad como un problema" a aquella que la visualiza creativamente como fuente de oportunidades para resolver 
parte de los problemas que la afectan, conociendo para ello su medioambiente, su clima y su comunidad.

Rueda (2013) reivindica la visión ecosistémica casi una década después, realzando el concepto de eficiencia en la organización de los seres vivos de la naturaleza, condición que la hace permanecer en el tiempo. En los sistemas urbanos, en tanto, la ecuación de eficiencia, expresada por el autor como E/nH -siendo E: energía requerida, n: número de personas jurídicas y $\mathrm{H}$ : diversidad de personas jurídicas o complejidad urbana- tiende a la ineficiencia. Esto se explica porque la variable E crece con el tiempo debido a la competencia de territorios por el consumo de recursos. Este autor postula, entonces, un urbanismo ecológico en el cual la variable E de la ecuación disminuya con el tiempo, alcanzando así la llamada sostenibilidad urbana. Los cuatro ejes de análisis desarrollados como marco conceptual son la compacidad en la estructura de tejidos urbanos, la complejidad en su organización, la eficiencia de su metabolismo y la cohesión social. Desde la perspectiva del metabolismo urbano, la habitabilidad presenta amplias acepciones, siendo una de ellas la habitabilidad urbana basada en la eficiencia energética de los edificios y energías renovables no convencionales. Por ejemplo, la orientación y anchura del edificio en función de ventilación cruzada, captación de aguas pluviales y captación solar.

En el sentido señalado, una zonificación territorial del clima adquiere importancia, porque está develando la localización de fuentes energéticas solares y eólicas, condiciones de pluviosidad y características mesoclimáticas, que sirvan de referencia para aproximarse a la escala local urbana, en la cual se determinan finalmente las necesidades energéticas de edificios y barrios para alcanzar su confort térmico. Algunos autores afirman que una organización espacial de las ciudades en que se integraran las características geoclimáticas de un territorio facilitaría una relación armónica con los ecosistemas naturales (McHarg, 1992). El reconocimiento de las condiciones climáticas locales de un territorio permite abordar la cuestión energética en la planificación urbana y ambiental, particularmente las demandas y ofertas energéticas necesarias para el acondicionamiento climático en las ciudades (Cárdenas e Higueras, 2015; Givoni, 1998; Marsh, 1998). Por tanto, se cree que una propuesta de biozonas en el territorio chileno se presenta como una cuestión relevante al desarrollo urbano sostenible, en un contexto de adaptación al cambio climático.

La actual Política Nacional de Desarrollo Urbano Chileno considera al territorio como un bien único e irrepetible y que, por tanto, debe regularse bajo el principio del bien común. Esto reivindica la necesidad de reconocer características peculiares del mismo para ser utilizadas como un recurso natural en la planificación y gestión territorial (Ministerio de Vivienda y Urbanismo [Minvu], 2014a). Por ejemplo, los diversos climas y subclimas reconocibles en un territorio podrían reunirse en áreas homogéneas. La política establece, entre sus objetivos principales, la calidad de vida de las personas y el desarrollo sustentable, aspectos que pretende concretar mediante políticas específicas por cada ámbito temático. En materias ambientales, se identifican problemas como la agresión a los sistemas naturales y se propone, en consecuencia, la integración de los ecosistemas a la planificación urbana. Una gestión eficiente de los recursos naturales, energéticos y de residuos es otro tópico relevante. Para ello, propone efectuar un monitoreo de variables ambientales y su 
medición mediante catastros. Esto sugiere propuestas de acción que validen información científica básica, de acceso público, para ser incorporada a la planificación de ciudades. Con ello se espera calibrar de mejor manera el uso de recursos climáticos en proyectos urbanos y de planificación en el territorio.

En este escenario, caben algunas preguntas de investigación: ¿cuál es el estado de las artes del urbanismo bioclimático desde la perspectiva del Estado chileno?; ¿qué instrumentos o herramientas estatales existen para abordar estas materias?; ¿es posible disponer, a nivel urbano, de información climática sistematizada para su utilidad en el urbanismo bioclimático?

Se cree que estas materias han sido abordadas tradicionalmente desde el Estado en forma sectorial, con instrumentos que tratan los subsistemas de agua, suelos, aire y energía, mas no de una forma integral. Se postula como hipótesis que transitar desde la planificación urbana hacia una planificación urbana-ambiental podría enfrentar de un modo holístico (ecosistémico) la cuestión ambiental en las ciudades, para avanzar hacia el desarrollo sustentable.

Dado que el clima afecta el funcionamiento de cada subsistema del urbanismo bioclimático, un elemento central y sustantivo en la integración de la visión ecosistémica a la planificación urbana-ambiental es conocer las condiciones climáticas a una escala local al interior de la ciudad. Actualmente existe en Chile información climática online a nivel territorial, la cual es provista por estaciones meteorológicas integradas en redes, pero tales instalaciones se sitúan principalmente en espacios extraurbanos o en aeropuertos -i.e. Agromet, Meteochile, cr2 (explorador.cr2.cl)- y resultan insuficientes para realizar estudios bioclimáticos al interior de la ciudad. De lo anterior se deduce la necesidad de disponer de herramientas espaciales, georreferenciadas, que entreguen información climática accesible, online y sensible a los planificadores urbanos, arquitectos, urbanistas, ingenieros y otros que toman decisiones sobre la ocupación del territorio y sus recursos naturales, en particular los recursos energéticos a escala urbana. Transitar desde una mesoescala a una escala local climática involucra mucho tiempo y recursos; disponer de valores aproximados en una unidad territorial menor que la actual norma chilena NCh1079 (zonificación climática) implica el acceso a información complementaria y un mejoramiento relativo frente a la ausencia de esa información en zonas intraurbanas.

El objetivo de este artículo es estudiar el estado de las artes del urbanismo bioclimático en Chile mediante la revisión de instrumentos o herramientas estatales que promuevan un acercamiento a estas materias. Como objetivo secundario, se quiere presentar una propuesta de unidades territoriales climáticas funcionales al urbanismo bioclimático, que faciliten el conocimiento de las condiciones ambientales y climáticas importantes para el confort humano y habitabilidad de los espacios, en diversas escalas de actuación. La metodología propuesta emplea bases de datos meteorológicas de cuarenta años obtenidas de estaciones localizadas en zonas rurales y urbanas periféricas (1960-2000). A partir de ellas, y con base en un Sistema de Información Geográfica (sIG), se delimitan biozonas para todo el territorio nacional, incluyendo tanto las áreas urbanizadas como aquellas que no lo están actualmente. La ventaja operativa de disponer de un SIG es la facilidad de consulta en archivos digitales, y desde cualquier lugar del territorio nacional, mediante internet, para la 
planificación de ciudades, y así hacer proyecciones frente a diversos escenarios de cambio climático (Ministerio del Medio Ambiente [MMA], 2016).

\section{Subsistemas del urbanismo bioclimático}

La comprensión de los ciclos de la naturaleza en coherencia con el diseño de las ciudades ha sido reivindicada desde la arquitectura del paisaje y la planificación ecológica (Ndubisi, 2014; Spirn, 1984). La interacción de los agentes abióticos, como el agua, el aire, los suelos y la energía, desde el urbanismo bioclimático (Sad de Assis \& Frota, 1999) constituye un subsistema que puede ser intervenido y regulado desde el Estado mediante instrumentos o herramientas.

La bioclimática per se es estimada principalmente por factores físicos meteorológicos (la radiación solar, el movimiento del aire, la temperatura y la humedad relativa) y humanos (el metabolismo y arropamiento), los cuales son modificados por la forma urbana, actividades humanas y edificaciones vecinas, creando una diversidad de microclimas y condiciones de confort local (Cárdenas Jirón, 2011; Olgyay \& Olgyay, 1962).

El análisis instrumental, sea de tipo normativo o técnico, vinculante a los subsistemas se presenta en la tabla 1 y tiene por objeto representar el estado de las artes en la materia. En ella se describen los instrumentos, características, escala, tipo de actuación y año para cada subsistema. Al final de cada subsección se indican las limitantes y oportunidades para avanzar hacia un urbanismo bioclimático en Chile. 


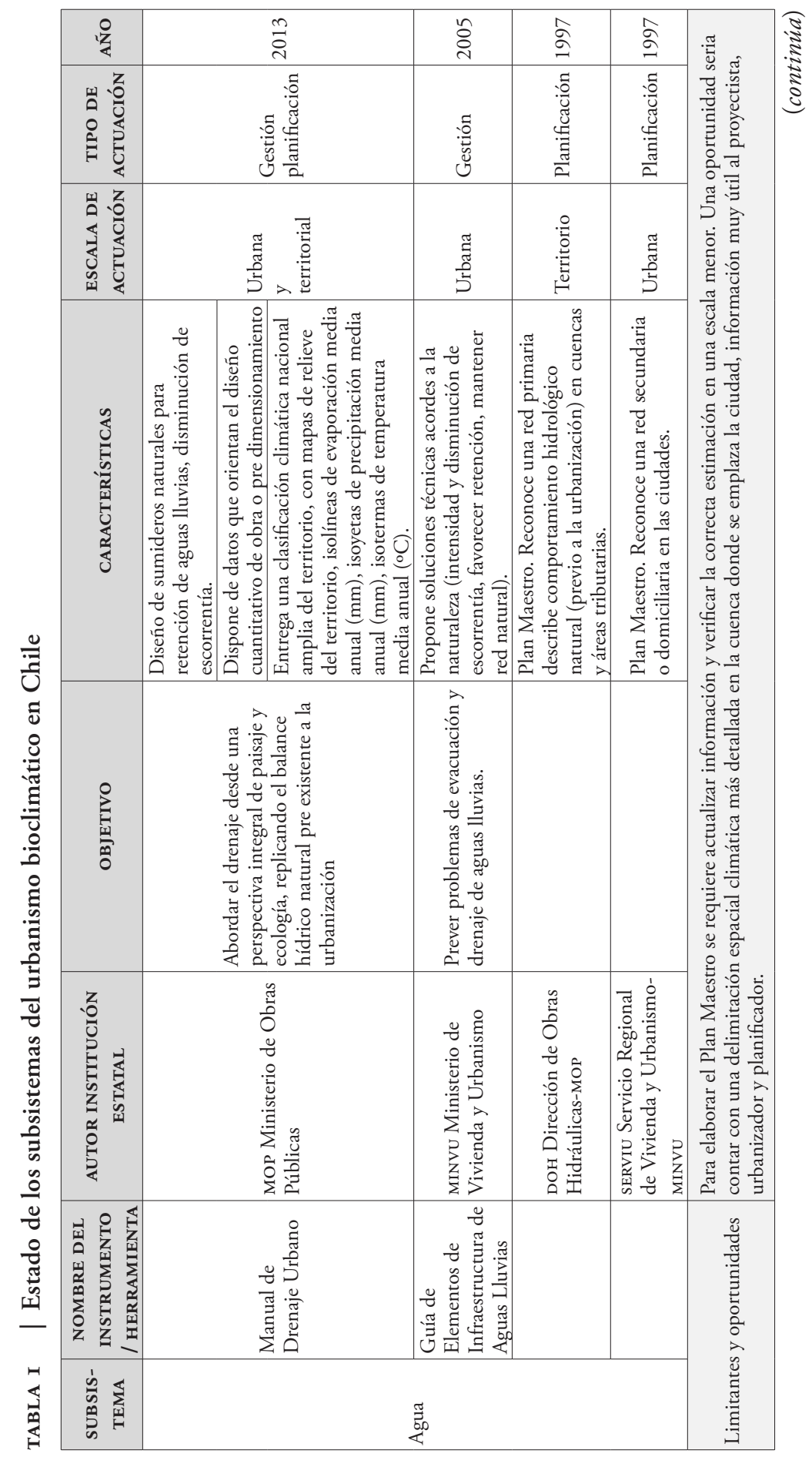




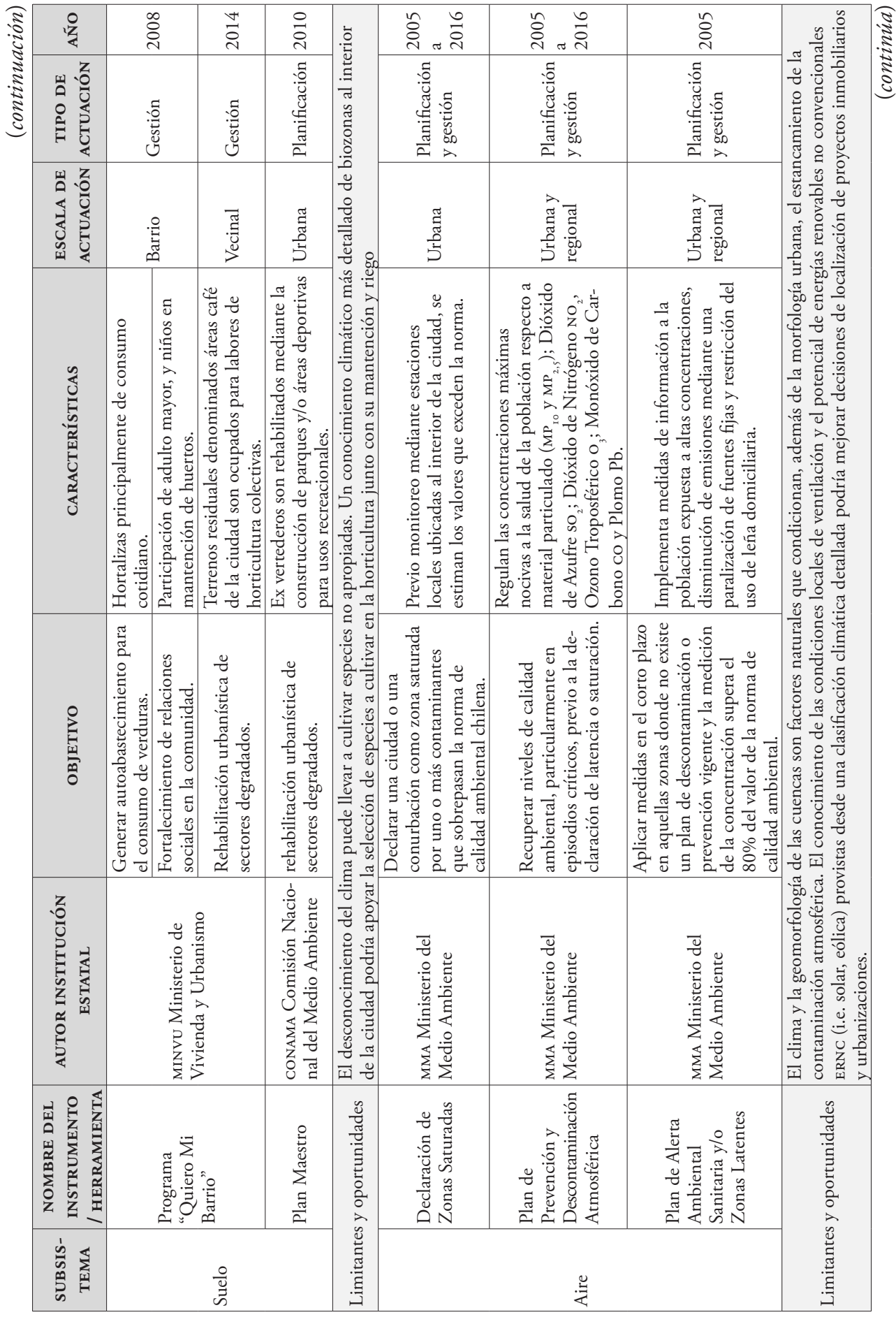




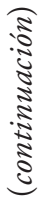

\begin{tabular}{|c|c|c|}
\hline 学 & $\begin{array}{l}\tilde{n} \\
\stackrel{\sim}{n}\end{array}$ & $\stackrel{\sim}{\vec{N}}$ \\
\hline 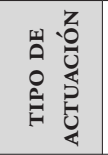 & & 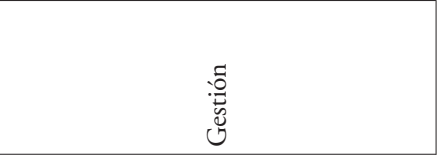 \\
\hline 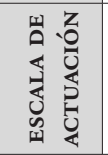 & 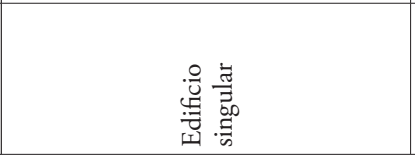 & 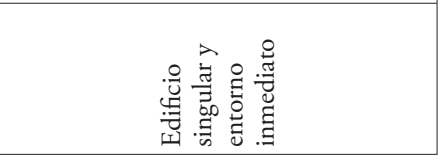 \\
\hline 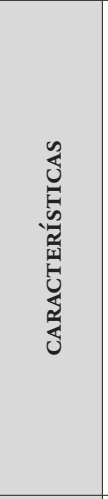 & 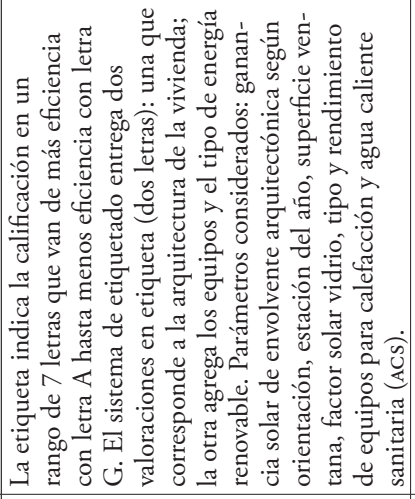 & 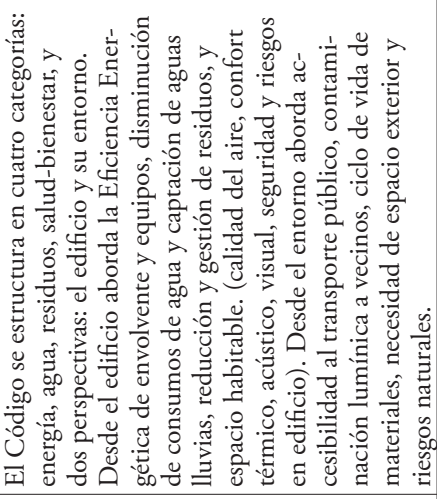 \\
\hline$\sum_{\substack{x \\
0}}^{0}$ & 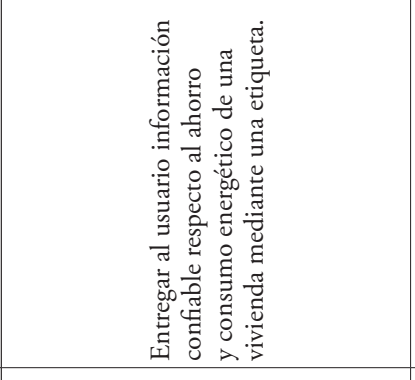 & 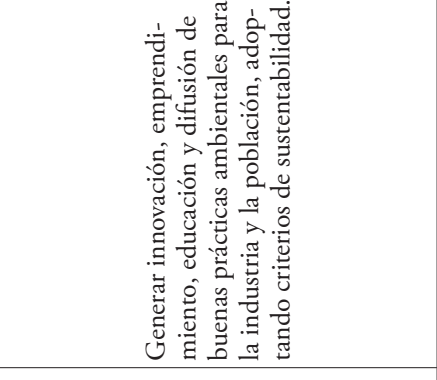 \\
\hline 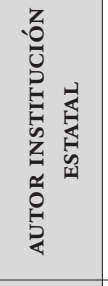 & 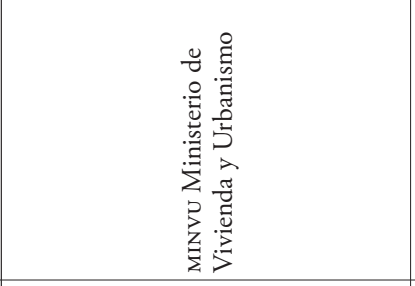 & 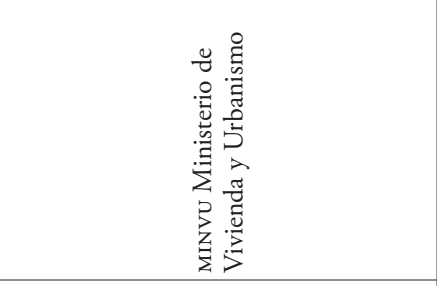 \\
\hline 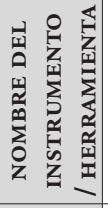 & 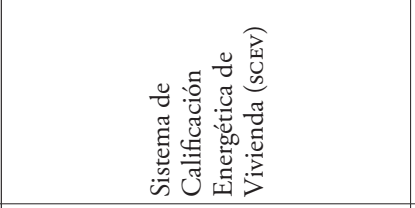 & 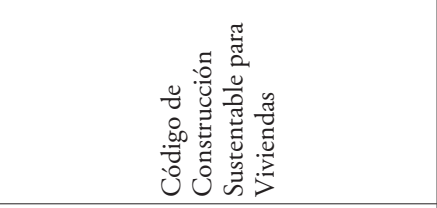 \\
\hline 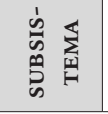 & 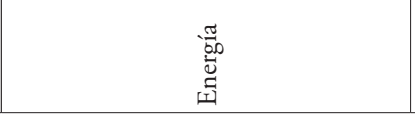 & \\
\hline
\end{tabular}




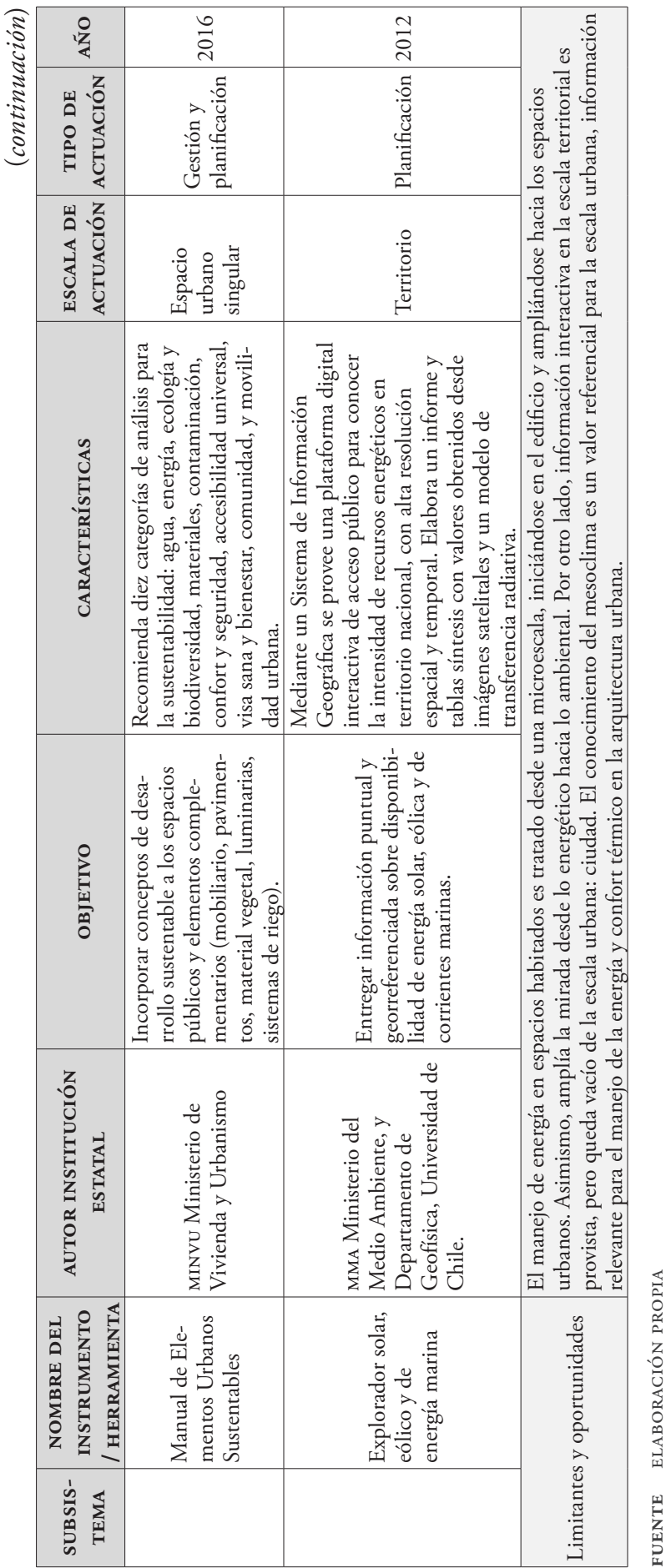




\section{El subsistema agua}

El recurso agua forma parte fundamental de la vida humana y es el componente principal del ciclo hidrológico que interactúa con el suelo y el aire. Las crecientes urbanizaciones sellan los suelos, reduciendo la capacidad de infiltración de las aguas. Con ello alteran la evapotranspiración de suelos, plantas y humedad ambiental de la atmósfera baja (Spirn, 1984), consideración que debe tenerse muy en cuenta en la planificación urbana y ambiental en Chile.

\section{El subsistema suelo}

El recurso suelo representa una oportunidad para revitalizar la ciudad. Los suelos y la vegetación constituyen elementos claves en el ciclo del carbono, por la descomposición de la materia orgánica; son el soporte territorial de la vida humana, animal y vegetal, que interactúa con el aire, el agua y el sol. El suelo cumple un rol fundamental para recibir y transformar los residuos orgánicos, tanto animales como vegetales, mediante un proceso natural de degradación. La horticultura ha desarrollado algunas técnicas para incidir en tal proceso: composting y bancal profundo. La técnica del composting consiste en la descomposición de la materia orgánica constituida por residuos vegetales, con la cual se forma una substancia oscura de tierra que enriquece el suelo, conocida como humus o compost. Al añadir esta substancia al jardín o parcelas para cultivos agrícolas, se producirá un mejor crecimiento en ellas.

La técnica del bancal profundo (raíz más larga que ancha) ha hecho posible cultivar en espacios reducidos, y ello ha permitido la generación de huertos urbanos en las ciudades. Por lo general estos son parcelas pequeńas, entre $25 \mathrm{~m}^{2}$ y $40 \mathrm{~m}^{2}$, en las cuales se cultivan los productos alimenticios. Las plantas cumplen una función primordial en el ciclo del carbono, porque mediante la fotosíntesis absorben anhídrido carbónico y eliminan oxígeno durante el día, proceso fundamental para la vida animal y humana, que se sustenta en la absorción de oxígeno y eliminación del anhídrido carbónico. Los vegetales son considerados sumideros de carbono, porque capturan el dióxido de carbono $\left(\mathrm{CO}_{2}\right)$ que circula permanentemente entre los suelos, los vegetales, la atmósfera y las aguas, cerrando el ciclo del carbono. Los animales, a su vez, eliminan residuos de los alimentos que no pueden asimilar y que se convierten en nutrientes para el suelo y plantas, circulando materia en los ciclos naturales del suelo. El nitrógeno, principal gas contenido en la atmosfera, es necesario para la vida de las plantas, fijándose en ellas naturalmente con la acción de las bacterias del tejido en descomposición o material orgánico putrefacto, que lo convierten en nitratos para enriquecer los suelos (Seymour, 2008).

Desde una perspectiva de barrio, se observan crecientemente redes de huertos urbanos localizados en espacios privados o públicos; en techumbres de edificios, en patios, aceras de calles, o simplemente en terrenos eriazos de uso colectivo o abandonado. La agricultura urbana emerge como una primera respuesta a los desafíos de seguridad alimentaria, principalmente al incidir en el autoabastecimiento y consumo de verduras (Miazzo, Minkjan \& Cities, 2013). Por otra parte, la regeneración de tejidos urbanos en obsolescencia a partir de la agricultura urbana, apoyada por comunidades de vecinos involucrados en redes sociales, constituye una acción revitalizadora en ambos tejidos, urbanos y sociales. En Chile se han incrementado 
los huertos urbanos en establecimientos educacionales, en comunidades de vecinos (Montecarmelo en Providencia, Barrio Modelo en San Ramón), en barrios urbanos patrimoniales (Yungay en Santiago Centro), y en recintos de reclusión y rehabilitación, en todos los cuales se organizan talleres y construyen eco barrios en la ciudad (Minvu, 2016a; sAu [Simposio de Agricultura Urbana], 2014)

\section{El subsistema aire}

El recurso aire es un componente fundamental del ciclo atmosférico y puede ser visto desde dos perspectivas: como un agente favorable a la ventilación de calles y espacios públicos, o como un agente patógeno propio de la ciudad. Las edificaciones en altura modifican la velocidad del viento en las corrientes advectivas, presentando diferentes gradientes de intensidad según sea la morfología urbana. Normalmente se reduce la intensidad con la altura de las edificaciones, aunque también podría intensificarse por corredores en cañones urbanos o en condiciones topográficas singulares (Fariña, 2001; Gandemer \& Guyot, 1981; Ng et al., 2011; Szucs, 2013).

El movimiento ascendente del aire influye en los intercambios convectivos de la atmósfera entre el palio urbano y la capa límite, renovando la masa de aire y dispersando los contaminantes (Landsberg, 1981; Oke, 1978). La radiación solar incidente sobre las superficies urbanas calienta las masas de aire en contacto con ellas (calles, edificios, estacionamientos, techumbres), y por convección se produce un ascenso del aire caliente hacia las capas superiores de la atmósfera, enfriándose en esta trayectoria. Estas masas de aire vuelven a bajar como aire frío cuando alcanzan un límite físico de altura, precipitando en forma de lluvia o bien como niebla que, al encontrase con partículas contaminantes, forma smog (contracción de smoke: humo y fog: niebla). Dadas las condiciones de inversión térmica en algunas cuencas, se produce una capa de mezcla contaminante, densa, que impide la dispersión de contaminantes por convección. Al ponerse el sol, se disipa el calor acumulado por el medioambiente construido, contribuyendo así a la isla de calor en la ciudad.

En Chile, el fenómeno de inversión térmica se asocia a una configuración geográfica confinada -normalmente cordones de cerros o altas montańas que rodean la ciudad- y también a condiciones climáticas, debido al predominio del Anticiclón Subtropical del Pacífico Suroriental, con cielos claros y despejados que ejercen su influencia en la región central chilena (Garreaud \& Rutlant, 2006). Particularmente, en el semestre invernal se producen bajas temperaturas y pocas precipitaciones, razón por la cual se forma un "techo" o capa superior de aire frío, denso, que impide la dispersión de contaminantes atmosféricos, porque inhibe el movimiento ascendente del aire. La capa de gases contaminados es transparente a la radiación ultravioleta y luz visible, pero opaca a la radiación infrarroja, atrapando así el calor y luego reemitiéndolo hacia las superficies de abajo en la ciudad. Con ello, altera el balance térmico terrestre y calidad de vida en las ciudades. La inversión térmica restringe las condiciones de ventilación de la cuenca de Santiago y otras ciudades chilenas que se ven afectadas durante el periodo invernal, condición que se supera cuando llega la primavera y cambian las temperaturas medias del aire, aumentando los vientos de subsidencia del aire. 
La altitud donde se sitúe la ciudad es también un factor relevante para la dilución de contaminantes. Por ejemplo, Valparaíso y Santiago están situadas en casi una misma latitud, pero con un espesor de la masa de aire distinto según se ubique la inversión térmica (figura 1). Si ella se ubica a 700 metros, Valparaíso tendrá unos 600 metros de espesor de masa de aire; en cambio Santiago, situado a unos $450 \mathrm{msnm}$, tendrá solo 250 metros de espesor de masa de aire para diluir los contaminantes (Pontificia Universidad Católica de Chile [PUC], 2001).

FIGURA I | Espesor relativo de masa atmosférica para dilución de contaminantes. Santiago en invierno

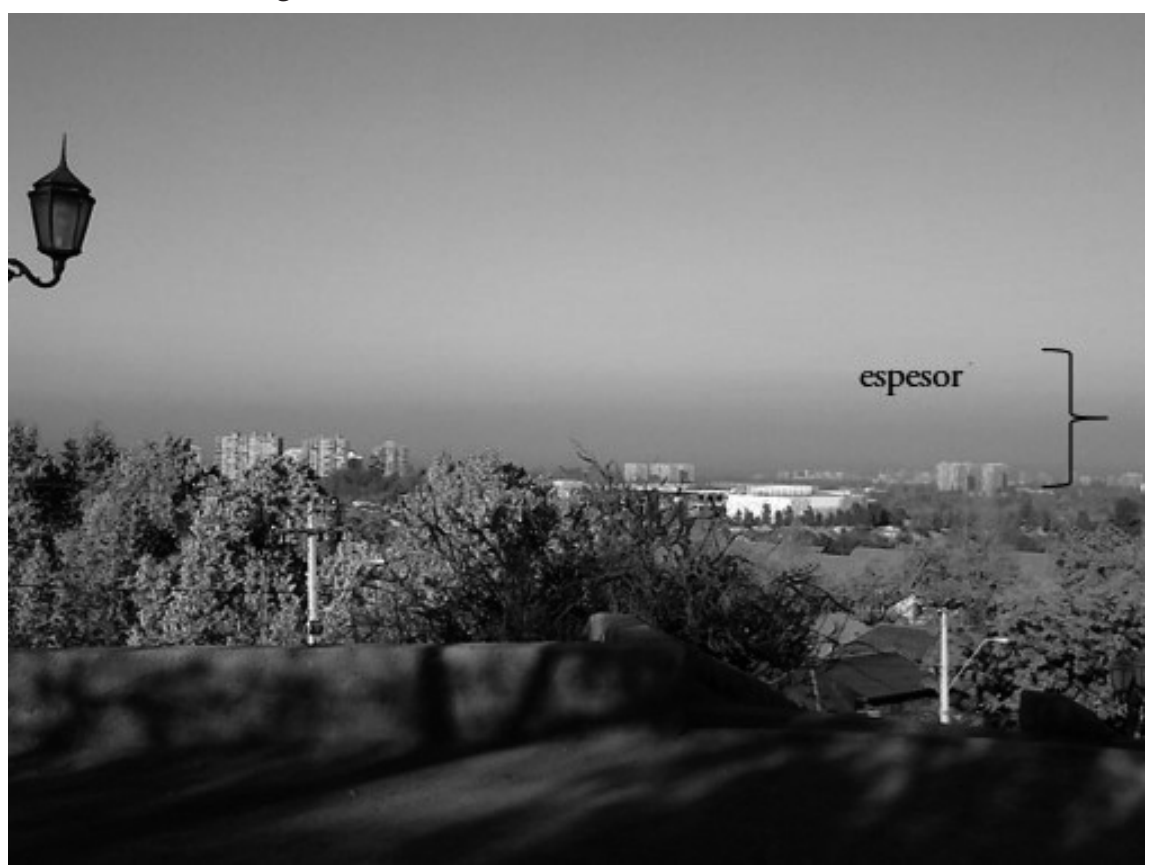

FUENTE ELABORACIÓN PROPIA

Desde el urbanismo, es muy importante conocer las características del viento local, brisas marinas y terrestres, vientos anabáticos (ascendentes) y catabáticos (descendentes), frecuencia, intensidad, orientación predominante, sentido de barlovento o sotavento, no solamente por la fuerza que ejerce sobre las construcciones, sino que por la capacidad de eliminación y difusión de contaminantes. Fariña (2001) advierte que las grandes tendencias del mesoclima no sirven para decisiones de emplazamiento de proyectos ni de diseño urbano, pues deben hacerse las correcciones necesarias para caracterizar el viento local. Por tanto, se advierte al lector que un mapa climático territorial de biozonas corresponde a una aproximación más desagregada que las clasificaciones preexistentes y su utilidad es una referencia aproximada para la planificación urbana. 


\section{El subsistema energía}

El aprovechamiento de las energías limpias es condición fundamental para el urbanismo bioclimático. El consumo energético basado en fuentes fósiles emite $\mathrm{CO}_{2}$, aumentando así la huella de carbono, lo que, junto con el agotamiento de dichas fuentes y elevación del precio, obliga a implementar energías renovables no convencionales (ERNC). Entre estas, la energía solar cobra gran relevancia por su disponibilidad en todo el planeta y, además, porque activa las otras fuentes de energía limpias: eólica, mareomotriz, hidroeléctrica, biomasa, exceptuando la geotérmica (Ministerio de Energía [ME], 2012; Sarmiento, 2007).

En Chile existen documentos donde se aboga por la eficiencia energética, principalmente en los sistemas constructivos de las edificaciones y alumbrado público de las calles. La concepción desde el urbanismo bioclimático no está suficientemente desarrollada, porque el interés se ha enfocado principalmente en el edificio-objeto, más que en las relaciones espaciales del edificio con su entorno urbano. No obstante, el organismo ministerial ha realizado avances sustantivos en vivienda e información de acceso público a la ciudadanía y simuladores online (Minvu, 2014b). Los documentos nacionales asociados a espacios habitados se indican en la tabla 1 .

Del análisis de los documentos se deduce la importancia de estimar el verdadero aporte solar según sea la localización de la vivienda en el territorio nacional, aporte influenciado por el comportamiento del clima y sus características peculiares de nubosidad, precipitaciones y vientos predominantes en un área geográfica dada. Una clasificación de áreas homogéneas según clima y geografía de las regiones más urbanizadas en Chile podría contribuir en la tarea de construir un sistema de calificación energética de la vivienda. No obstante, debe tenerse presente que condiciones morfológicas del entorno urbano al edificio podrían alterar sustancialmente el aporte solar a una vivienda, y con ello modificar su calificación energética.

A modo de crítica, se puede comentar, en primer lugar, que el Código de Construcción Sustentable para Viviendas mencionado en la tabla 1 representa un paso significativo en Chile. Por una parte, podría darle continuidad al Sistema de Calificación Energética de las Viviendas (SCEV); y por otra, podría ampliar el enfoque centrado solo en la energía, hacia uno ambiental que incluya otros aspectos propios de habitar la vivienda. Sin embargo, las nueve zonas climáticas empleadas por el Código y basadas en la norma chilena (NCh1079.Of2008) parecen insuficientes para caracterizar la diversidad climática de una localidad urbana.

Se sugiere que una diferenciación más desagregada de las áreas geográficas según climas homogéneos posibilitaría una caracterización mejor y, con ello, las recomendaciones de diseño pasivo pertinentes. Por otro lado, se propone un análisis de la transversalidad climática del territorio geográfico chileno en el eje cordillera a mar, dado que muestra desempeños bioclimáticos distintos para una misma zona geográfica. Gradientes de humedad relativa y nubosidad afectan significativamente el aporte solar y, por ende, la variación de su intensidad a lo largo del día, aspectos que debieran ser reconocidos por el Código. Igualmente, las brisas y vientos son parámetros útiles para el acondicionamiento ambiental y varían según las áreas geográficas al interior de una misma región, características que no tienen un comportamiento climático homogéneo en la región administrativa chilena. 
El reconocimiento de los diversos climas chilenos es fundamental para poder planificar, diseñar y proyectar ciudades. El Manual de elementos urbanos sustentables (Minvu, 2016a) reconoce la importancia del clima por su influencia en el uso de los espacios públicos, durabilidad de los materiales, vínculos con los servicios ecosistémicos; y sobre esa base recomienda una selección de los elementos urbanos constituyentes de un espacio público. El Manual aborda el concepto de sustentabilidad, aplicado mediante guías metodológicas y ejemplos prácticos, e incorpora en sus categorías de análisis gran parte de los conceptos fundamentales del urbanismo bioclimático.

El Ministerio de Energía mantiene en su plataforma web documentos de acceso abierto de gran relevancia para determinar fuentes energéticas renovables no convencionales (solar, eólica y mareomotriz) y planificar su uso (ME, 2012; 2017). Dada su resolución espacial, tales documentos son apropiados para estudios de generación energética de plantas a una mesoescala. Parámetros generales como la pluviosidad, la humedad relativa, la radiación solar, la temperatura del aire, los vientos, son necesarios para aproximarse a una caracterización del territorio a escala regional y local, de manera previa a la planificación. Así se hace posible transitar desde una planificación urbana hacia una planificación urbano-ambiental del territorio. Las propuestas de los instrumentos de planificación territorial (IPT), al igual que proyectos urbanos en consonancia con las características geográficas y climáticas del lugar donde se emplazan, permitirán avanzar hacia un urbanismo bioclimático $y$, con ello, propender hacia la ciudad sustentable.

\section{Clasificaciones nacionales de clima y de condiciones térmicas en Chile}

La norma chilena (NCh1079.Of2008) entrega una clasificación de nueve zonas climáticas para todo el territorio nacional, denominadas: Norte Litoral, Norte Desértica, Norte Valles Transversales, Central Litoral, Central Interior, Sur Litoral, Sur Interior, Sur Extremo y Andina (Instituto Nacional de Normalización [INN], 2010) (figura 2). Esta clasificación fue elaborada el año 1977 y actualizada en 2008, treinta ańos después, lo cual aporta una versión al día y útil para que el proyectista la incorpore en el diseño arquitectónico. El documento entrega un glosario de definiciones relativas al clima para una precisa comprensión e interpretación de cada término.

Por otra parte, existe una zonificación térmica presentada en el Manual de aplicación de la reglamentación térmica (Minvu, 2006), a partir de la cual se establecen requisitos de acondicionamiento térmico a las viviendas para los complejos de techumbre, muros y pisos ventilados, además de superficies máximas de ventanas. El Manual provee exigencias para disponer de confort higrotérmico al interior de las viviendas y con ello garantizar mínimas condiciones de habitabilidad. La reglamentación regula la demanda de energía en los edificios mediante la eficiencia energética de la envolvente; emplea y optimiza las ganancias internas y externas, además de promover sistemas no contaminantes, eficientes y de bajo costo. Divide el territorio nacional en siete zonas térmicas según grados-día de calefacción con base en $15^{\circ} \mathrm{C}$ y fija coeficientes de transmitancia térmica y resistencia térmica en techumbres, muros y pisos ventilados. 
FIGURA 2 | Zonificación climática chilena según Nch1079.of2008
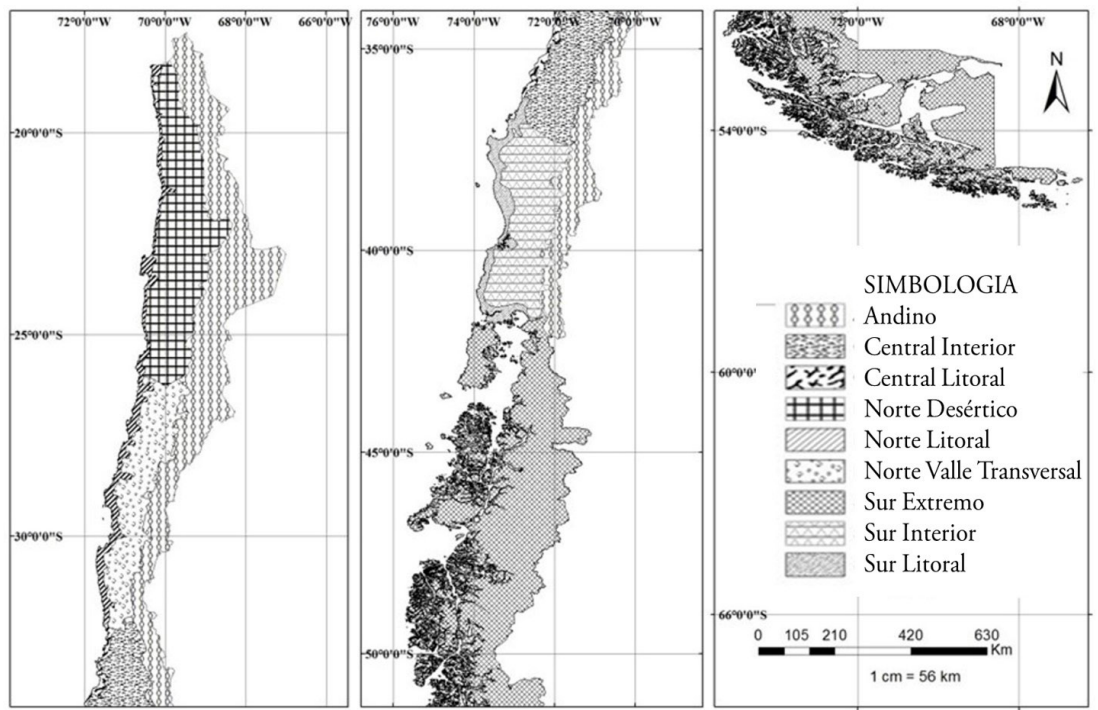

FUENTE PREPARADO POR DANIELA SANTEliCES A PARTIR DE BASE NORMATIVA DEL INN (2OIO), NCHI $079.0 F 2008$

Adicionalmente, en Chile se han realizado trabajos importantes en relación con zonificaciones bioclimáticas del territorio; entre ellos se puede citar Bioclimatología de Chile (Di Castri \& Hajek, 1976), Geografía de los climas (Romero, 1985), Mapa agroclimático de Chile (Novoa et al., 1989), Sinopsis bioclimática y vegetacional de Chile (Luebert \& Pliscoff, 2006) y Atlas bioclimático de Chile (Uribe, Cabrera, De la Fuente \& Paneque, 2012). Todas estas iniciativas tenían objetivos distintos, como su uso en agricultura (Novoa et al., 1989; Uribe et al., 2012) y vegetación (Di Castri \& Hajek, 1976; Luebert \& Pliscoff, 2006). Estas zonificaciones se basaron en diferentes metodológicas específicas, las primeras de las cuales -temporalmente hablando- se basaban en el trazado de isolíneas para cada variable y análisis visual de mapas. Las más modernas, en cambio, se basan en procesamiento digital y el uso de complejas ecuaciones topoclimáticas para establecer la variabilidad espacial de una variable; estas se agrupan en zonas mediante análisis de conglomerados y/o en algoritmos de segmentación espacial (Morales, 1997; Morales et al., 2009). Todas estas zonificaciones, a pesar de ser muy valiosas, adolecen del enfoque necesario para ser aplicadas en su totalidad a la arquitectura y el urbanismo bioclimático.

Por lo anteriormente señalado, se realiza una proposición de zonificación climática para las ciudades chilenas considerando variables climáticas similares a las usadas para definir la norma chilena, pero se desagregan espacialmente en unidades menores, arrojando como resultado una clasificación basada en zonas climáticas de mayor detalle que las actuales. Se cree que esta agregación podría reducir el margen de error de valores macroclimáticos, dada la resolución espacial menor en la definición de áreas climáticas homogéneas. Las variables empleadas por la norma 
chilena fueron temperatura (media mensual, y oscilación media mensual), insolación (Wh/m²/día), soleamiento (horas sol/día), humedad relativa (\%), nubosidad (décimas), precipitación mensual $(\mathrm{mm})$, vientos predominantes, y heladas.

En esta propuesta de clasificación se ha incorporado también el concepto de grados-día con base en $15{ }^{\circ} \mathrm{C}$, integrado como un layer adicional que permita estimar posteriormente el confort térmico en espacios y las necesidades de calefacción. Este umbral de confort es un referente solamente, dado que se debe considerar las condiciones de microclima urbano al interior de las ciudades, condiciones producidas por la espacialidad de la morfología urbana, el albedo de los materiales, la vegetación, las características de la atmósfera y la topografía local, el perfil de horizonte, todos aspectos que modifican la temperatura local estimada.

\section{Materiales y método}

Área de estudio

El presente estudio se realizó en Chile continental, ubicado en el Cono Sur de América entre los paralelos $17^{\circ} 30^{\prime}$ y $56^{\circ} 30^{\prime}$ de Latitud Sur alineado en torno a los $70^{\circ}$ de Longitud Oeste. Su ubicación entre el océano Pacífico y la cordillera de Los Andes le confiere una particular configuración, siendo el país más largo del mundo, con $4.329 \mathrm{~km}$ de longitud (figura 3). En su longitud se manifiesta casi la totalidad de climas presentes en el planeta, los cuales, según la clasificación climática de Köppen, son: clima desértico en el norte; tundra y glaciares en el este y el sur; húmedo subtropical en Isla de Pascua; mediterráneo en Chile central, oceánico en el sur y el polar en el Territorio Chileno Antártico.

FIGURA 3 | Área de estudio correspondiente a Chile continental

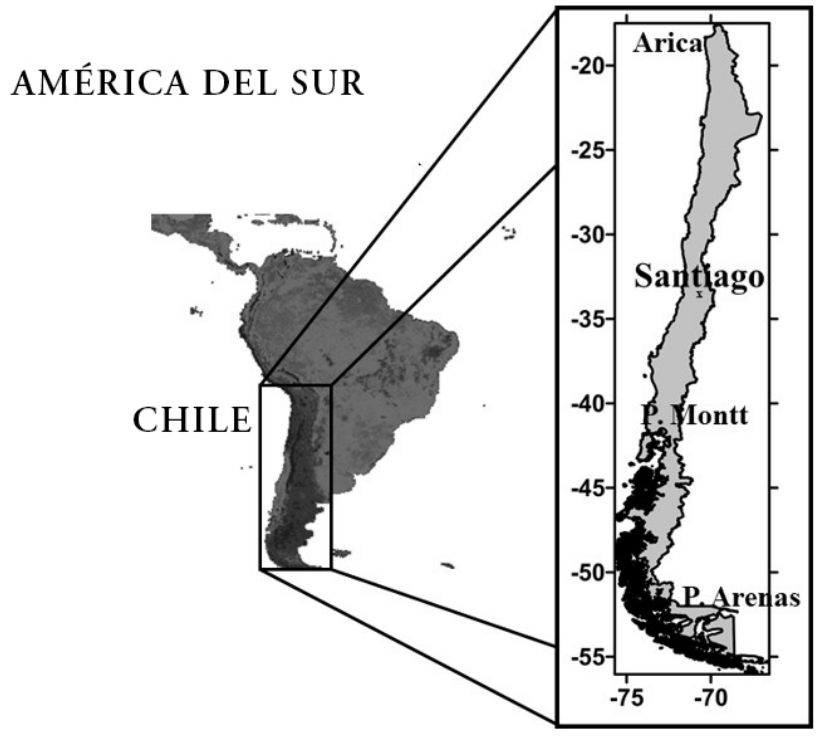

FUENTE ELABORACIÓN PROPIA 


\section{Datos utilizados}

Se utilizaron las capas ambientales proporcionadas por Worldclim (Hijmans et al., 2005), que corresponden a variables climatológicas de temperaturas máximas, mínimas y medias, en valores medios mensuales (http://biogeo.berkeley.edu/ Worldclim/Worldclim.htm). Worldclim es una base de datos climatológica en formato matricial o raster; sus datos fueron generados por interpolación espacial a partir de promedios mensuales de más de 46.000 estaciones meteorológicas en todo el mundo en el período 1950-2000, y un modelo de elevación (MDE). El MDE corresponde a una estructura matricial de datos que representa la distribución espacial de la altitud. Worldclim utilizó el MDE de distribución gratuita de resolución espacial de $1000 \mathrm{~m}$, llamado GTOPO3O. Este MDE provee datos de altitud en metros sobre el nivel del mar para todo el mundo en coordenadas geográficas, latitud y longitud, y referenciado a wGS64 (Us Geological Survey [USGs], 1997).

Para la validación de los datos Worldclim a nivel nacional, se utilizaron datos de la red de estaciones meteorológicas históricas con a lo menos diez o más años de mediciones, las cuales fueron recolectadas de: Climatología en Chile (Programa de las Naciones Unidas para el Desarrollo [PNUD] / Gobierno de Chile, 1964), Atlas Agroclimático de Chile (Instituto de Investigaciones Agropecuarias [INIA], 1989), Dirección General de Aguas (DGA, 1987) y anuarios de la Dirección Meteorológica de Chile (DMC). En total se recopilaron 1478 estaciones con datos medios mensuales, pero fue necesaria la homogeneización de los datos recopilados, debido fundamentalmente a que las estaciones eran provenientes de distintas fuentes de información y presentaron sistemas de referencia diferentes, por lo que toda la información de carácter geográfico fue reproyectada y trabajada en el Datum wGs64 en coordenadas esféricas.

\section{Variables climáticas}

Para cuantificar la cantidad de calor acumulado o pérdida por el medioambiente urbano, se utilizan los días grado acumulados sobre un umbral, que nos dan una aproximación al número de días en que se necesita usar aire acondicionado. En el mundo, este tipo de índices es utilizado para lograr un mejoramiento en las condiciones energéticas y de habitabilidad mediante el diseño arquitectónico. Los días grado corresponden a la resta entre la temperatura media diaria y una temperatura característica usada como umbral, que normalmente oscila entre $25^{\circ} \mathrm{C}$ o $18{ }^{\circ} \mathrm{C}$, según sea el tipo de cálculo. Estas unidades de calor se acumulan cuando la diferencia anterior es mayor que cero, lo que se realiza durante todo el año, dando un número característico para cada localidad. El mismo concepto es aplicable cuando esta diferencia es negativa, suma anual que puede asociarse con las necesidades de calefacción. Este tipo de estudios asociados a las necesidades de calefacción y refrigeración por medio del índice de días grado no es nuevo y es ampliamente utilizado (Thom, 1952). Se calculó, además, la amplitud u oscilación térmica (diferencia entre la temperatura máxima y mínima diaria) media mensual, el número de heladas anuales y temperatura mínima absoluta como indicadores de la severidad climática de un lugar (Abramowitz \& Stegun, 1964; Morales, 1997; Snedecor \& Cocharn, 1979). 
La radiación solar global es estimada a partir de los datos de temperatura de Worldclim, utilizando el método propuesto por Bristow y Campbell (1984), quienes desarrollaron un algoritmo empírico para la estimación de la radiación solar global diaria en función de las temperaturas máximas y mínimas de aire (Allen, Pereira, Raes \& Smith, 1998; Iqbal, 1986; Samani, 2000; Supit \& Van Kappel, 1998). Este método ha sido aplicado con buenos resultados en todo el mundo, y en Chile, por varios autores para la estimación de la radiación solar global; sin embargo, han tenido que calibrar el método a las condiciones locales (Aburto, 2007; Álvarez, Mitasova \& Allen, 2011; Ayllón, 2012; Castellvi, 2001; Castillo \& Santibañez, 1981; Da Silva, Da Silva, Alves Júnior \& Carvalho, 2012; Frauberth et al., 2015; Grant, et al., 2004; Meza \& Varas, 2000; Morales et al., 2009; Queiroz et al., 2014).

\section{Clasificación bioclimática}

La clasificación bioclimática consiste en la división del territorio en áreas más pequeñas, homogéneas espacialmente. Su caracterización está fundamentalmente basada en aspectos físicos, como son el clima y sus factores determinantes (Qiyao, Jingming \& Baopu, 1991). Este proceso se llevó a cabo mediante la aplicación de un análisis de clúster utilizando el algoritmo k-medias, el cual se basa en la distancia euclidiana como medida de cuantificación de la similitud, para la clasificación automática de datos en grupos homogéneos previamente desconocidos (Pérez, 2004). Los objetos corresponden a cada uno de los elementos ordenados en una matriz (imagen), llamados píxeles (picture element). Las variables meteorológicas usadas para calcular las áreas homogéneas fueron: temperatura máxima media mensual de enero y julio (TXE у TXJ, respectivamente); temperatura mínima media mensual de enero y julio (TNE y TNJ, respectivamente); amplitud térmica de enero y julio (ATE y ATJ, respectivamente); precipitación media anual (PPMA); radiación solar global media mensual de enero y julio (RSGE y RSGJ, respectivamente); días grado anuales con base térmica de $15^{\circ} \mathrm{C}$ (DGAI 5); temperatura mínima absoluta de enero y julio (TMAE y TMAJ, respectivamente); y número de heladas anuales ( $\mathrm{NHA}$ ); evapotranspiración potencial de enero y julio (ETPE y ETPJ, respectivamente); humedad relativa de enero y julio (HRE y HRJ); e intensidad media vientos de enero y julio (ve y VJ). Finalmente, las propiedades asociadas a ese elemento se almacenan en un arreglo vectorial, cuyos elementos corresponden a cada una de las variables climatológicas consideradas. El resultado de este proceso es una segmentación del espacio en áreas homogéneas con iguales características bioclimáticas, las cuales son transformadas en un formato vectorial shape de ArcGis. Esta nueva estructura de almacenamiento permite la consulta por un usuario a través de un sistema de información geográfica.

\section{Resultados}

La revisión del marco conceptual del urbanismo bioclimático, junto con el análisis de los instrumentos técnicos chilenos afines, entregó las bases para conocer el estado del arte en la materia a nivel nacional. A partir de ello, se observó la necesidad de contar con un instrumento de zonificación climática de alcance nacional, operativo y accesible a los profesionales que toman decisiones sobre la construcción actual de la ciudad, y la planificación urbana y ambiental del territorio. 
Con tal fin se desarrolló un programa computacional destinado a implementar los algoritmos de estimación, usando para ello Visual Basic Excel y, como insumo principal, las variables climatológicas aportadas por los datos Worldclim. A partir de este programa, se calcularon la amplitud térmica, los días grado anuales con base en $15^{\circ} \mathrm{C}$ y $18{ }^{\circ} \mathrm{C}$, la temperatura mínima absoluta, el número de heladas anuales y la radiación global anual. Todas las variables se almacenaron en un archivo binario compatible con cualquier sistema de información geográfica. Estos datos se encontrarán disponibles en la página web www.lares.uchile.cl, desde donde es posible descargarlos llenando previamente un formulario de registro. Los datos proporcionados en esta base de datos pueden ser utilizados con fines académicos; sin embargo, los investigadores que los usen deben explicitar la cita en las referencias del artículo científico respectivo. Los datos se encuentran disponibles en el formato raster GEOTIFF, de tal forma que su uso sea fácil y manejable en cualquier sistema de información geográfica, ya sea de uso público o con licencia privada.

FIGURA 4 Zonificación bioclimática del territorio continental chileno separado en norte, centro y sur austral

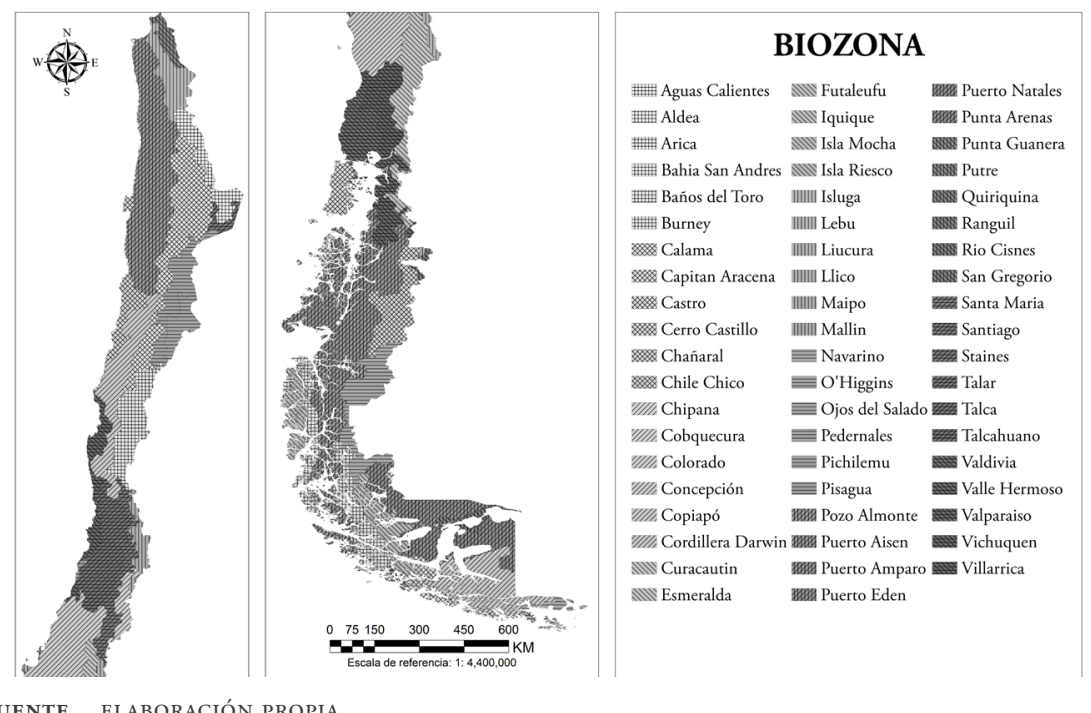

FUENTE ELABORACIÓN PROPIA

La figura 4 muestra una segmentación del espacio en zonas bioclimáticas homogéneas para Chile mediante análisis de clúster por el método k-medias. Se muestran los 43 distritos mesoclimáticos homogéneos obtenidos, los cuales establecen la variación espacial de las características climáticas del área de estudio, según la resolución espacial de trabajo. Cada una de las unidades mesoclimáticas homogéneas incluidas en la figura posee valores medios asociados de las variables climatológicas con las cuales se construyeron los clústeres, los cuales son mostrados como una tabla de datos anexa. La principal utilidad de las zonas mesoclimáticas es que pueden ser usadas mediante sistemas de información geográficas (GIS) para ser integradas en 
las aproximaciones al conocimiento de la bioclimatología en un territorio; además, esta integración es relativamente fácil y a partir de ella se pueden generar en menor tiempo resultados satisfactorios para la planificación urbana.

Las características climáticas promedio de cada biozona se detallan en la tabla 2. Esta muestra los valores medios de las variables climatológicas para cada zona bioclimática definida en la figura 4, donde los nombres de cada zona se asignaron por la localidad más importante en ella. En la tabla 2 las variables climatológicas se representan por acrónimos, donde TNOI y TXOI corresponden a las temperaturas mínimas y máximas de enero respectivamente; en cambio TNO7 y TXO7 corresponden a las temperaturas mínimas y máximas de julio, respectivamente, todas en grados Celcius. De igual forma se interpretan HR para humedad relativa (\%), RG para radiación global $\left(\mathrm{MJ} / \mathrm{m}^{2}\right.$ día $)$ y, finalmente, PPMA para la precipitación media anual ( $\mathrm{mm}$ ), donde 01 corresponde al mes de enero y 07 al mes de julio, lo cual se interpreta similarmente para cada variable. Los valores climatológicos asociados a cada biozona son consultados en la tabla 2 y pueden ser identificados en la figura 4 . Adicionalmente, estos valores pueden ser consultados en forma dinámica a partir del archivo disponible para descargar en KMZ o sHApe de ArcGis en la página web del Laboratorio de Investigación en Ciencias Ambientales lares (www.lares.uchile. cl), de la Facultad de Ciencias Agronómicas de la Universidad de Chile. Si no se desea descargar, es posible consultarla en tiempo real en el explorador diseńado para su consulta. Las condiciones climáticas locales de cada biozona pueden ser consultadas a partir de este mismo navegador, donde se presentan valores medios, máximos y mínimos; sin embargo, es necesario considerar el efecto de la isla térmica en ciudades para contar con valores más aproximados a la realidad.

TABLA 2 Valores medios de algunas de las variables climatológicas para cada zona bioclimática definida en la figura 4

\begin{tabular}{|l|r|r|c|c|c|c|c|c|r|}
\hline $\begin{array}{c}\text { ZONA } \\
\text { CLIMÁTICA }\end{array}$ & TNOI & TNO7 & TXOI & TXO7 & HROI & HRO7 & RGOI & RGO7 & PPMA \\
\hline Putre & $-2,5$ & $-10,3$ & 13,5 & 8,5 & 48,0 & 32,9 & 26,4 & 17,8 & 286,4 \\
\hline Aguas Calientes & $-0,4$ & $-9,3$ & 15,7 & 7,9 & 41,9 & 27,1 & 27,0 & 15,7 & 58,1 \\
\hline Isluga & 2,7 & $-5,5$ & 18,1 & 11,6 & 52,8 & 43,1 & 26,0 & 16,6 & 123,9 \\
\hline Pozo Almonte & 13,1 & 5,9 & 25,0 & 17,6 & 66,3 & 69,7 & 23,2 & 12,8 & 6,0 \\
\hline Calama & 7,0 & $-0,7$ & 20,7 & 13,5 & 53,6 & 48,3 & 25,0 & 13,6 & 26,1 \\
\hline Talar & 1,6 & $-7,6$ & 17,4 & 9,3 & 38,6 & 24,7 & 26,9 & 15,0 & 50,0 \\
\hline Chańaral & 15,4 & 8,2 & 25,1 & 17,5 & 72,2 & 81,3 & 21,1 & 10,4 & 20,3 \\
\hline Chipana & 17,6 & 11,2 & 25,7 & 17,8 & 71,4 & 80,4 & 18,7 & 9,6 & 1,2 \\
\hline Iquique & 16,9 & 11,7 & 25,1 & 17,7 & 75,5 & 86,7 & 19,0 & 9,5 & 1,3 \\
\hline Punta Guanera & 16,7 & 11,4 & 25,1 & 17,8 & 75,4 & 86,2 & 19,3 & 10,0 & 1,0 \\
\hline Pisagua & 15,8 & 10,2 & 24,3 & 17,2 & 72,8 & 83,1 & 19,1 & 10,4 & 0,6 \\
\hline Arica & 17,1 & 10,8 & 25,6 & 18,2 & 75,2 & 85,9 & 19,3 & 11,1 & 1,4 \\
\hline Ojos del Salado & $-0,8$ & $-6,9$ & 11,6 & 5,1 & 43,1 & 27,2 & 23,9 & 11,7 & 70,8 \\
\hline Pedernales & 2,5 & $-3,7$ & 15,1 & 8,9 & 47,6 & 35,5 & 24,0 & 12,3 & 35,5 \\
\hline Copiapó & 11,3 & 4,1 & 23,0 & 15,0 & 68,7 & 74,7 & 23,4 & 10,6 & 76,2 \\
\hline Bańos del Toro & 1,4 & $-5,6$ & 14,3 & 5,9 & 55,3 & 48,3 & 24,6 & 10,6 & 120,1 \\
\hline
\end{tabular}


(continuación)

\begin{tabular}{|c|c|c|c|c|c|c|c|c|c|}
\hline $\begin{array}{c}\text { ZONA } \\
\text { CLIMÁTICA }\end{array}$ & TNOI & TNO7 & TXOI & TX07 & HROI & HRO7 & RGOI & RGO7 & PPMA \\
\hline Valparaíso & 13,5 & 6,3 & 24,5 & 15,4 & 74,0 & 84,3 & 22,7 & 9,0 & 256,1 \\
\hline Maipo & $-0,8$ & $-9,1$ & 14,5 & 1,5 & 50,9 & 41,9 & 26,9 & 9,1 & 496,9 \\
\hline Llico & 9,4 & 5,6 & 22,2 & 12,9 & 74,9 & 86,2 & 24,6 & 6,6 & $1.188,8$ \\
\hline Santa María & 7,5 & 4,6 & 16,3 & 9,9 & 54,5 & 63,2 & 17,4 & 4,8 & 632,0 \\
\hline Talcahuano & 10,2 & 5,3 & 21,9 & 12,3 & 72,3 & 83,8 & 22,9 & 6,4 & 939,0 \\
\hline Quiriquina & 5,5 & 3,0 & 10,6 & 6,4 & 38,0 & 44,0 & 11,1 & 3,3 & 443,7 \\
\hline Cobquecura & 11,0 & 5,4 & 22,3 & 12,3 & 73,1 & 84,1 & 22,8 & 6,6 & 925,9 \\
\hline Vichuquén & 11,5 & 4,2 & 26,8 & 12,4 & 74,8 & 86,0 & 26,9 & 7,6 & 827,5 \\
\hline Pichilemu & 14,2 & 5,8 & 28,0 & 13,7 & 74,9 & 85,9 & 25,6 & 7,7 & 610,9 \\
\hline Talca & 12,3 & 3,7 & 28,3 & 13,7 & 70,4 & 79,2 & 27,6 & 8,8 & 540,3 \\
\hline Concepción & 10,0 & 3,4 & 25,7 & 11,3 & 71,4 & 81,8 & 27,3 & 6,9 & $1.236,1$ \\
\hline Colorado & 3,2 & $-5,3$ & 20,3 & 4,8 & 55,2 & 52,6 & 28,5 & 8,3 & 793,5 \\
\hline Curacautín & 5,3 & 0,3 & 23,0 & 6,7 & 62,3 & 67,5 & 28,9 & 5,9 & $1.685,0$ \\
\hline Santiago & 6,2 & $-2,2$ & 23,1 & 8,0 & 60,7 & 62,1 & 28,3 & 8,7 & 766,0 \\
\hline Isla Mocha & 7,3 & 4,6 & 18,1 & 10,5 & 61,4 & 70,9 & 20,7 & 5,2 & $1.026,4$ \\
\hline Lebu & 8,3 & 4,8 & 20,3 & 11,4 & 66,9 & 77,1 & 22,5 & 5,9 & $1.329,1$ \\
\hline Ranguil & 8,8 & 5,3 & 20,8 & 12,1 & 69,8 & 80,4 & 22,9 & 6,1 & $1.191,6$ \\
\hline Valdivia & 9,0 & 3,8 & 18,8 & 9,4 & 70,9 & 81,6 & 21,1 & 4,9 & $1.783,9$ \\
\hline Futaleufú & 4,8 & $-2,3$ & 17,7 & 4,2 & 60,6 & 64,9 & 24,6 & 5,1 & $1.608,8$ \\
\hline Mallín & 5,8 & $-2,9$ & 17,6 & 3,8 & 54,2 & 59,5 & 23,5 & 4,6 & 650,5 \\
\hline Valle Hermoso & 4,3 & $-3,8$ & 16,2 & 2,9 & 54,6 & 58,0 & 23,6 & 4,8 & 982,8 \\
\hline Liucura & 3,4 & $-1,9$ & 22,3 & 4,9 & 55,6 & 56,7 & 29,8 & 6,2 & $1.096,3$ \\
\hline Villarrica & 7,9 & 1,3 & 20,3 & 7,8 & 66,7 & 75,3 & 24,2 & 5,3 & $2.066,4$ \\
\hline Río Cisnes & 4,8 & $-3,9$ & 16,4 & 2,7 & 53,5 & 57,9 & 23,2 & 4,5 & 561,1 \\
\hline Bahía San Andrés & 7,4 & 2,9 & 12,5 & 6,9 & 71,1 & 80,0 & 15,2 & 3,1 & $2.088,0$ \\
\hline Castro & 9,2 & 4,0 & 14,9 & 8,0 & 68,7 & 79,2 & 15,9 & 3,7 & $2.034,7$ \\
\hline Puerto Amparo & 7,5 & 1,9 & 13,6 & 6,0 & 64,1 & 73,5 & 16,1 & 3,3 & $2.259,9$ \\
\hline Chile Chico & 5,1 & $-3,3$ & 14,8 & 2,7 & 58,8 & 65,1 & 21,2 & 4,0 & 843,3 \\
\hline Puerto Aisén & 5,4 & $-1,7$ & 13,7 & 3,6 & 61,4 & 67,5 & 19,5 & 3,6 & $1.721,2$ \\
\hline O’Higgins & 3,2 & $-4,4$ & 12,5 & 2,0 & 59,9 & 64,7 & 20,7 & 3,4 & $1.153,7$ \\
\hline Esmeralda & 4,4 & 1,1 & 8,2 & 3,9 & 47,3 & 54,4 & 10,7 & 1,8 & $3.213,6$ \\
\hline Cerro Castillo & 3,6 & $-2,2$ & 10,4 & 2,7 & 39,9 & 46,2 & 14,1 & 2,1 & 254,0 \\
\hline Aldea & 4,4 & 0,8 & 8,5 & 3,8 & 48,5 & 55,6 & 11,3 & 1,9 & $2.710,1$ \\
\hline San Gregorio & 6,1 & $-0,5$ & 14,8 & 4,6 & 54,6 & 64,7 & 19,3 & 2,3 & 278,3 \\
\hline Puerto Edén & 4,2 & 0,1 & 8,9 & 3,5 & 46,9 & 53,7 & 12,1 & 1,9 & $2.114,0$ \\
\hline Puerto Natales & 5,1 & $-2,2$ & 14,4 & 3,8 & 58,1 & 67,4 & 20,0 & 2,6 & 486,9 \\
\hline Isla Riesco & 3,6 & $-1,9$ & 10,6 & 2,9 & 52,4 & 60,0 & 15,9 & 2,0 & $1.002,7$ \\
\hline Burney & 3,7 & $-1,2$ & 9,6 & 3,0 & 51,5 & 58,7 & 14,2 & 1,9 & $1.567,3$ \\
\hline \begin{tabular}{|l|} 
Cordillera \\
Darwin \\
\end{tabular} & 2,7 & $-2,3$ & 9,9 & 2,4 & 57,2 & 65,2 & 16,7 & 1,8 & 705,7 \\
\hline Navarino & 1,9 & $-0,9$ & 6,0 & 1,9 & 32,7 & 38,1 & 9,4 & 1,0 & 296,5 \\
\hline Punta Arenas & 5,4 & $-0,7$ & 13,6 & 4,1 & 56,1 & 65,8 & 18,8 & 2,2 & 378,2 \\
\hline Staines & 2,0 & $-0,3$ & 5,0 & 1,7 & 28,9 & 33,4 & 7,3 & 0,8 & 709,1 \\
\hline Capitán Aracena & 2,6 & $-0,8$ & 7,3 & 2,3 & 42,9 & 49,4 & 11,4 & 1,2 & 761,9 \\
\hline
\end{tabular}

FUENTE ELABORACIÓN PROPIA 


\section{Discusión}

Desde el punto de vista bioclimático, es posible separar el territorio nacional continental en cuatro grandes zonas, las cuales se explican en primera aproximación por el gradiente latitudinal: i) La Zona Norte, caracterizada por un clima árido a semiárido, con un marcado gradiente desde la costa y hacia la cordillera de los Andes, definiendo tres franjas climáticas diferentes, las cuales se encuentran asociadas a la zona litoral, el desierto propiamente tal, y la zona andina. ii) La Zona Central, con un marcado clima templado, caracterizado por inviernos cortos de entre cuatro a seis meses y con época estival sin precipitaciones, además de presentar una marcada diferenciación entre la costa, el valle interior y la cordillera de los Andes. iii) La Zona Sur, con un clima templado lluvioso, inviernos más largos, pero con un comportamiento diferenciado entre litoral y cordillera de los Andes. iv) Finalmente, la Zona Austral hasta Tierra del Fuego presenta un clima más frío, dividido en un área más lluviosa y otra estepárica, caracterizada por poseer una menor precipitación (Romero, 1985). Esta breve descripción se complementa por la variabilidad mesoclimática que se observa en Chile, la cual es representada cuantitativamente por las diferentes biozonas mostradas en la figura 4. Cada una de las biozonas posee características particulares desde el punto de vista bioclimático, y en general se encuentran definidas principalmente por sus variables climatológicas, las cuales se describen en la tabla 2.

\section{Conclusiones}

La información generada en el presente trabajo puede ser mejorada y actualizada en la medida en que se disponga de más información, sobre todo al densificar la red nacional de estaciones meteorológicas y homogeneizar la tecnología de las mismas. Este hecho es aún más relevante si consideramos la baja distribución espacial de estaciones meteorológicas al interior de las ciudades chilenas.

El estudio del urbanismo bioclimático y el estado del arte en Chile mediante el análisis de instrumentos técnicos aplicados, refleja aproximaciones sectoriales en los subsistemas analizados: el agua, los suelos, el aire y la energía. No obstante, el análisis de la gestión del subsistema agua integra visiones ecosistémicas con el territorio, lo cual indica una mejora relativa en su implementación respecto a los otros subsistemas.

El método de zonificación aplicado permitió la identificación de varias biozonas con características específicas, definidas fundamentalmente a partir de la precipitación y la temperatura, usando índices bioclimáticos como los grados-día. De esta forma, es posible utilizar esta información generada para complementar la existente dada por la norma chilena NCh1079.Of2008.

Es importante mencionar que para aplicar esta información en los cálculos asociados al consumo energético de viviendas en ciudades, es necesario tomar en cuenta el efecto de la isla térmica o de calor urbana (ICU). En efecto, varios estudios a nivel nacional muestran que en la época estival ella puede ser varios grados por sobre el entorno más rural. Asimismo, se deberá considerar el efecto de cuerpos de 
agua y concentraciones de vegetación en parques para ajustar los valores referenciales provistos.

La propuesta de zonificación climática territorial enfocada hacia el bienestar térmico humano, a diferencia del enfoque de productividad agrícola o de biodiversidad precedente, representaría un avance gradual al conocimiento requerido para iniciar aplicaciones bioclimáticas en la ciudad o villorrios.

El método descrito, a pesar de su sencillez, incluye el análisis de estadística espacial, y ha demostrado ser robusto en la estimación del campo térmico asociado con la acumulación de grados-día. La literatura actual presenta varios métodos de estimación espacial de datos meteorológicos; sin embargo, es posible, de una forma sencilla, obtener mapas de campos climatológicos en forma continua y minimizar el error de estimación.

El acceso online de variables meteorológicas y la agregación en unidades espaciales menores pueden disminuir el margen de error al momento de estimar la temperatura medioambiente y demandas energéticas en edificios. Asimismo, el conocimiento del resto de las variables climáticas locales facilita una caracterización geoclimática de menor resolución espacial que las zonificaciones existentes en Chile.

La propuesta constituye una aproximación territorial para el urbanismo bioclimático; no obstante, se requiere ajustar las variables climáticas de la cartografía entregada, a la morfología urbana de cada asentamiento humano. La configuración tridimensional de las ciudades o forma urbis crea microclimas y características peculiares necesarias de considerar en los instrumentos de planificación territorial (IPT). La contribución de esta propuesta permite discurrir desde una planificación urbana hacia una planificación ambiental del territorio.

\section{Agradecimientos}

Se agradece el financiamiento otorgado por los proyectos de investigación Fondecyt No 1161-809 y Fondecyt No 1130-139, Conicyt. Adicionalmente, se reconoce con gratitud las observaciones y sugerencias de los evaluadores de la revista, que contribuyeron a mejorar esta publicación. Se agradece el apoyo cartográfico de la estudiante Daniela Santelices V., Facultad de Ciencias Agronómicas, Universidad de Chile.

\section{Referencias bibliográficas}

Abramowitz M. \& Stegun, I. A. (eds.) (1964). Handbook of Mathematical Functions (décima edición). Applied Mathematics Series 55. Washington, D.c.: National Bureau of Standards (NBS). http://people.math.sfu.ca/ -cbm/aands/abramowitz_and_stegun.pdf

Aburto Schweitzer, C. (2007). Elaboración de un modelo de estimación de la distribución espacial de la radiación solar global mensual para Chile central. Memoria de título de Ingeniería en Recursos Naturales Renovables. Universidad de Chile, Santiago, Chile. http:// www.tesis.uchile.cl/tesis/uchile/2007/aburto_c/pdf/aburto_c.pdf 
Álvarez J., Mitasova, H. \& Allen, H. (2011). Estimating monthly solar radiation in southcentral Chile. Chilean Journal of Agricultural Research, 71(4), 601-609. http://dx.doi. org/10.4067/S0718-58392011000400016

Allen, R., Pereira, L., Raes D. \& Smith, M. (1998). Crop evapotranspiration (guidelines for computing crop water requirements). FAO Drainage and Irrigation Paper 56. Rome: Food and Agriculture Organization. http://www.agraria.unirc.it/documentazione/ materiale_didattico/1462_2016_412_24101.pdf

Ayllón, V. (2012). Comparación de métodos de estimación de la radiación solar en Maracay, Venezuela. Agronomía Tropical, 62(1-4), 137-144. https://docplayer.es/9841546Comparacion-de-metodos-de-estimacion-de-la-radiacion-solar-en-maracay-venezuela.html

Bettini, V. (1998). Elementos de Ecología Urbana. Serie Medio Ambiente. Madrid: Editorial Trotta.

Bristow, K. L. \& Campbell, G. S. (1984). On the relationship between incoming solar radiation and daily maximum and minimum temperature. Agricultural and Forest Meteorology, 31(2), 159-166. https://doi.org/10.1016/0168-1923(84)90017-0

Cárdenas Jirón, L. A. (2011) Caracterización de patrones bioclimáticos en tejidos urbanos residenciales. Tesis doctoral. Universidad Politécnica de Madrid, España.

Cárdenas Jirón, L. A. \& Higueras, E. (2015). El barrio solar. Madrid: Mairea.

Castellvi, F. (2001). A new simple method for estimating monthly and daily solar radiation. Performance and comparison with other methods at Lleida (NE Spain); a semiarid climate. Theoretical and Applied Climatology, 69(3-4), 231-238. https://doi. org/10.1007/s007040170028

Castillo, H. \& Santibáñez, F. (1981). Evaluación de la radiación solar global y luminosidad en Chile I. Calibración de fórmulas para estimar radiación solar global diaria. Agricultura Técnica, 41(3), 145-152.

Comisión Nacional del Medio Ambiente (Conama), Chile (2010). Primer reporte del manejo de residuos sólidos en Chile. Santiago, Chile: Conama. http://www.hidronor.cl/ pdf/1_Primer_Reporte_del_Manejo_de_Residuos_S\%C3\%B3lidos_en_Chile_ Conama_2010.pdf

Da Silva, C., Da Silva, V. J., Alves Júnior, J. \& Carvalho, H. (2012). Radiação solar estimada com base na temperatura do ar para três regiôes de Minas Gerais. Revista Brasileira de Engenharia Agricola e Ambiental, 16(3), 281-288. http://doi.org/10.1590/S141543662012000300008

Di Castri, F., \& Hajek, E. R. (1976). Bioclimatología de Chile. Santiago, Chile: Vicerrectoría Académica, P. Universidad Católica de Chile.

Dirección General de Aguas (DGA), Ministerio de Obras Públicas (MOP), Chile (1987). Balance hidrico de Chile. Santiago, Chile: Dirección General de Aguas, Ministerio de Obras Públicas.

Dirección Meteorológica de Chile (DMC) (varios años). Anuarios Climatológicos. En línea: https://climatologia.meteochile.gob.cl/application/index/anuarios

EMB Construcción (2015, mayo). Canalización de aguas lluvia. EMB Construcción, 11(153), 3738. http://www.microbyte.cl/cons/flipbook/201505/\#/37 
Espinace, J. (2009). Rehabilitación de sitios degradados: lodos, una alternativa a implementar. Seminario Tratamiento Sustentable de Lodos. Embajada de Suecia, abril 2009. Valparaíso: Pontificia Universidad Católica de Valparaíso, Grupo de Geotecnia. http:// icc.ucv.cl/geotecnia (Diapositivas en https://slidex.tips/download/rehabilitacion-desitios-degradados-lodos-una-alternativa-a-implementar)

Fariña Tojo, J. (2001). La ciudad y el medio natural. Madrid: Akal.

Frauberth Camayo-Lapa, B., Massipe-Hernández, J. R., Pomachagua-Paucar, J. E., TorresTen, A. \& Quispe-Flores, M. O. (2015). Desarrollo del modelo bristow campbell para estimar la radiación solar global de la región de Junín, Perú. Tecnología Química, 35(2), 220-234. http://scielo.sld.cu/scielo.php?script=sci_arttext\&pid=S2224$61852015000200008 \& \operatorname{lng}=\mathrm{es} \& \mathrm{t} \operatorname{lng}=\mathrm{es}$

Gandemer, J. \& Guyot, A. (1981). La protection contre le vent: aerodynamique des brise-vent et conseils pratiques. París: Centre scientifique et technique du batiment.

Garreaud, R. \& Rutlant, J. (2006) Factores meteorológicos de la contaminación atmosférica. En R. Morales (ed.), Contaminación atmosférica urbana. Episodios criticos de contaminación ambiental en la ciudad de Santiago (pp. 35-54). Santiago, Chile: Editorial Universitaria.

Girardet, H. (1996) The GaIA Atlas of Cities. New directions for sustainable urban living. Londres: Gaia Books.

Girardet, H. (2004) Cities people planet: liveable cities for a sustainable world. Londres: Earthscan.

Givoni, B. (1998). Climate considerations in building and urban design. Nueva York: John Wiley $\&$ Sons.

Grant, R. H., Hollinger, S. E., Hubbard, K. G., Hoogenboom, G. R. \& Vanderlip, L. (2004). Ability to predict daily solar radiation values from interpolated climate records for use in crop simulation models. Agricultural and Forest Meteorology, 127(1), 65-75. http:// doi.org/10.1016/j.agrformet.2004.07.016

Higueras, E. (1998). Urbanismo bioclimático. Criterios medioambientales en la ordenación de asentamientos. Cuadernos de Investigación Urbanística (CIUR), 24. Madrid: Instituto Juan de Herrera.

Higueras, E. (2006) Urbanismo bioclimático. Barcelona: Editorial GG.

Hijmans, R., Cameron, S., Parra, J., Jones, P. \& Jarvis, A. (2005). Very high resolution interpolated climate surfaces for global land areas. International Journal of Climatology, 25(15), 1965-1978. http://doi.org/10.1002/joc.1276

Instituto Nacional de Normalización (INN), Chile (2010). Arquitecturay construcción. Zonificación climático habitacional para Chile y recomendaciones para el diseño arquitectónico. Norma Chilena Oficial NCh1079.Of2008. Santiago: Instituto Nacional de Normalización.

Iqbal, M. (1983). An introduction to solar radiation. Toronto: Academic Press.

Landsberg, H. E. (1981). The urban climate. Nueva York/Londres: Academic Press.

Luebert, F. \& Pliscoff, P. (2006). Sinopsis bioclimática y vegetacional de Chile. Santiago: Editorial Universitaria.

Marsh W. M. (1998). Landscape Planning: Environmental Applications. UsA: John Wiley \& Sons. McHarg, I. (1992). Design with Nature. Nueva York: John Wiley \& Sons.

Mertens, E. (1999). Bioclimate and city planning - open space planning. Atmospheric Environment, 33(24-25), 4.115-4.123. https://doi.org/10.1016/S1352-2310(99)00153-3 
Meza, F. \& Varas, E. (2000). Estimation of mean monthly solar global radiation as a function of temperature. Agricultural and Forest Meteorology, 100(2-3), 231-241. https://doi. org/10.1016/S0168-1923(99)00090-8

Miazzo, F., Minkjan, M. \& Cities. (2013). Farming the city: food as a tool for today's urbanization. Haarlem, Holanda: Valiz/Trancity.

Ministerio de Energía (ME), Chile (2012). Explorador del recurso solar en Chile. Documentación y manual de uso. Santiago, Chile: Ministerio de Energía. http://walker.dgf.uchile.cl/ Explorador/Solar2/info/Documentacion_Explorador_Solar.pdf

Ministerio de Energía (ME), Chile (2017) Energías renovables. Santiago, Chile: Ministerio de Energía. http://www.energia.gob.cl/energias-renovables

Ministerio de Medio Ambiente (MMA), Chile (2014). Planes de Descontaminación Atmosférica. Estrategia 2014-2018. Santiago, Chile: MMA. http://portal.mma.gob.cl/planes-dedescontaminacion-atmosferica-estrategia-2014-2018/

Ministerio de Medio Ambiente (мма), Chile (2016). Plan de Acción Nacional de Cambio Climático 2017-2022. Departamento de Cambio Climático. http://portal.mma.gob.cl/ plan-de-accion-nacional-de-cambio-climatico-2017-2022-pancc-ii/

Ministerio de Obras Públicas (мор), Chile (2013). Manual de drenaje urbano. Santiago, Chile: MOP. http://www.doh.gov.cl/manualdrenajeurbano/Paginas/default.aspx

Ministerio de Vivienda y Urbanismo (Minvu), Chile (2005). Guía de diseño y especificaciones de elementos urbanos de infraestructura de aguas lluvias. Santiago, Chile: Minvu. http:// www.minvu.cl/opensite_20090529093818.aspx

Ministerio de Vivienda y Urbanismo (Minvu), Chile (2006). Manual de aplicación reglamentación térmica. Ordenanza General de Urbanismo y Construcciones Artículo 4.1.10. Santiago, Chile: Minvu. http://www.minvu.cl/opensite_20070417155724.aspx

Ministerio de Vivienda y Urbanismo (Minvu), Chile (2014a). Politica Nacional de Desarrollo Urbano. Ciudades sustentables y calidad de vida. Santiago, Chile: Minvu. http://cndu. gob.cl/wp-content/uploads/2014/10/L4-Politica-Nacional-Urbana.pdf

Ministerio de Vivienda y Urbanismo (Minvu), Chile (2014b). Manual de usuario de herramienta web. Sistema de Calificación Energética de Viviendas en Chile. Santiago, Chile: Minvu. http://calificacionenergetica.minvu.cl/media/Manual_herramienta_web_Final.pdf

Ministerio de Vivienda y Urbanismo (Minvu), Chile (2014c). Código de construcción sustentable para viviendas, Chile. Santiago, Chile: Minvu. http://csustentable.minvu.cl/wpcontent/uploads/2015/09/C\%C3\%B3digo-de-Construcci\%C3\%B3n-SustentablePrimera-Versi\%C3\%B3n-espa\%C3\%B1ol.pdf

Ministerio de Vivienda y Urbanismo (Minvu), Chile (2015). Sistema de Calificación Energética de Viviendas en Chile. Santiago, Chile: Minvu. http://csustentable.minvu.cl/item/ codigo-de-construccion-sustentable/

Ministerio de Vivienda y Urbanismo (Minvu), Chile (2016a). Manual de elementos urbanos sustentables. Santiago, Chile: Minvu.

Ministerio de Vivienda y Urbanismo (Minvu), Chile (2016b). Compilado de buenas prácticas barriales. Programa de Recuperación de Barrios. "Quiero Mi Barrio". Santiago, Chile: Minvu.

Morales, L. (1997). Evaluación y zonificación de riesgo de heladas mediante modelización topoclimática. Tesis doctoral, Universidad de Concepción, Chile. 
Morales, L., Castellaro, G., Parra, J. C., Espinosa, J., Lang, F., Ojeda, N. \& Soto, N. (2009). Método de generación de cartografía climática usando regresiones con pesos geográficos. Revista Simiente, 79(1-2), 74-82. http://www.sach.cl/revista/pdf/ simiente_79_1_2.pdf

Ndubisi, F. (2002). Ecological planning: a historical and comparative synthesis. Baltimore: Johns Hopkins University Press.

Ng, E., Yuan, C., Chen, L., Ren, C., \& Fung, J. C. H. (2011). Improving the wind environment in high-density cities by understanding urban morphology and surface roughness: A study in Hong Kong. Landscape and Urban Planning, 101(1), 59-74.0. https://doi. org/10.1016/j.landurbplan.2011.01.004

Novoa, R., Villaseca, S., Del Canto, P., Rouanet, J., Sierra, C. \& Del Pozo, A. (1989). Mapa agroclimático de Chile. Santiago, Chile: Instituto de Investigaciones Agropecuarias.

Oke, T. R. (1978). Boundary layer climates. Londres: Methuen.

Olgyay, V. \& Olgyay, A. (1962). Design with climate: Bioclimatic approach to architectural regionalism. Londres: Princeton University Press.

Pérez, C. (2004). Técnicas de análisis multivariante de datos, Aplicaciones con SPSS. Madrid: Pearson Educación.

Pontificia Universidad Católica de Chile (PUC). (2001) Altitud, inversión térmica y contaminación. http://www7.uc.cl/sw_educ/contam/efect/efur06.htm

Programa de las Naciones Unidas para el Desarrollo (PNUD)-Gobierno de Chile (1964). Proyecto Hidrometeorológico. Climatología en Chile. Fascículo I. Valores normales de 36 estaciones seleccionadas. Período 1916-1945. Santiago, Chile: s.e.

Qiyao, L., Jingming, Y. \& Baopu, F. (1991). A method of agrotopoclimatic division and its practice in China. International Journal of Climatology, 11(1), 86-96. https://doi. org/10.1002/joc.3370110107

Romero, H. (1985). Geografía de los climas de Chile. Tomo xi: Colección de Geografía de Chile. Santiago, Chile: Instituto Geográfico Militar (IGM).

Rueda, S. (2013). El urbanismo ecológico. Urban-e 02, 1-22. http://urban-e.aq.upm.es/ articulos/ver/el-urbanismo-ecol-gico

Sad de Assis, E. \& Barros Frota, A. (1999). Urban bioclimatic design strategies for a tropical city. Atmospheric Environment, 33(24-25), 4135-4142. https://doi.org/10.1016/ S1352-2310(99)00155-7

Samani, Z. (2000). Estimating solar radiation and evapotranspiration using minimum climatological data. Journal of Irrigation and Drainage Engineering, 126(4), 265-267. https://doi.org/10.1061/(ASCE)0733-9437(2000)126:4(265)

Sarmiento, P. (2007) Energía solar en arquitectura y construcción. Santiago: RIL Editores.

SAU - Primer Simposio de Agricultura Urbana SAU 3 (2014). Traduciendo el zumbido del enjambre. Hacia una comprensión del estado actual de la Agricultura Urbana en Chile. Actas del Primer Simposio de Agricultura Urbana saur 3. Santiago, Chile: Editorial cU. http://cultivosurbanos.cl/investigacion/libro-sau-2013-traduciendo-el-zumbidodel-enjambre/

Seymour, J. (2008). The new self-sufficient gardener. Londres: Dorling Kindersley.

Snedecor C. \& Cocharn, G. (1979). Métodos Estadísticos. México, D.F.: Cecsa (Compañia Editorial Continente).

Spirn, A. W. (1984). The granite garden: urban nature and human design. Nueva York: Basic Books. 
Supit, I. \& Kappel, R. R. van (1998). A simple method to estimate global radiation. Solar Energy, 63(3), 147-160. https://doi.org/10.1016/S0038-092X(98)00068-1

Szucs, A. (2013). Wind comfort in a public urban space. Case study within Dublin Docklands. Frontiers of Architectural Research, 2(1), 50-66. https://doi.org/10.1016/j. foar.2012.12.002

Thom, H. C. S. (1952). Seasonal degree-day statistics for the United States. Monthly Weather Review, 80(9), 143-147. https://doi.org/10.1175/1520-0493(1952)080\%3C0143:S DSFTU\%3E2.0.CO;2

U.s. Geological Survey (1997). Publications of the U.S. Geological Survey, 1996. Washington, D.C.: United States Government Printing Office. https://pubs.usgs.gov/ unnumbered/70043742/report.pdf

Universidad de Chile \& Fundación para la Innovación Agraria (2017). Atlas Agroclimático de Chile. Estado actual y tendencias del clima. 6 Tomos. Santiago, Chile: Universidad de Chile / Fundación para la Innovación Agraria. [Tomo I: Regiones de Arica y Parinacota, Tarapacá y Antofagasta http://www.agrimed.cl/atlas/tomo1.html; Tomo II: Regiones de Atacama y Coquimbo http://www.agrimed.cl/atlas/tomo2.html; Tomo III: Regiones de Valparaíso, Metropolitana, O’Higgins y Maule http://www.agrimed.cl/ atlas/tomo3.html; Tomo rv: Regiones del Bío Bío y La Araucanía http://www.agrimed. cl/atlas/tomo4.html; Tomo v: Regiones de Los Ríos y Los Lagos http://www.agrimed. cl/atlas/tomo5.html; Tomo vi: Regiones de Aysén y Magallanes http://www.agrimed. $\mathrm{cl} /$ atlas/tomo6.html]

Unwin, R. (1909). Town planning in practice. Londres: Adelphi Terrace.

Uribe, J. M., Cabrera, R., De la Fuente, A. \& Paneque, M. (2012). Atlas bioclimático de Chile. Santiago, Chile: Universidad de Chile. 